\title{
Analysis of electrical activity in healthy and dystrophic muscle in man
}

\author{
R. G. WILLISON \\ From The Institute of Neurology, Queen Square, London
}

In clinical electromyography it is often difficult to analyse the differences between the electrical activity of healthy and of dystrophic muscle. The measurement of the duration and amplitude of single motor unit potentials is perhaps the most reliable method of quantitative assessment in current use (Kugelberg, 1949; Pinelli and Buchthal, 1953). For this purpose it is necessary to record the waveforms of individual motor units at levels of voluntary effort so low that they may be seen in isolation. Unfortunately these measurements do not characterize one of the most striking features of the activity of dystrophic muscles, which is the appearance of large numbers of potentials at relatively low tensions.

Another approach involves the analysis of the pattern of activity during strong contraction when it is no longer possible to identify single motor unit potentials on the trace. The only technique in current use for this purpose is frequency analysis (Richardson, 1951; Walton, 1952) but even this has not gained wide acceptance, and subjective assessment of the electromyograph (E.M.G.) by eye and ear is still current practice in the diagnosis of muscle disease. Subjective methods are not only undesirable in the diagnosis of established cases (Gilliatt, 1962) but are even more unsuitable for the detection of potential carriers of inherited muscular dystrophy in whom the abnormalities may be expected to be slight (Barwick, 1963; van den Bosch, 1963). The first step away from this situation is to describe the pattern seen during voluntary effort in simple numerical terms. In order to do this a method has been developed which involves the analysis of voluntary activity recorded with a concentric needle electrode at standard tensions; photographic records are measured by a counting device in order to obtain the number of potential changes per second present in the trace and the mean amplitude of these potentials. The details of the counting method have been previously described (Willison, 1963) but a summary is given below.

\section{MATERIAL AND METHODS}

Observations were made on 24 control subjects. They were healthy subjects associated with the laboratory, $\bar{C}$ their ages ranging from 18 to 40 years. Twenty patients with muscular dystrophy and polymyositis were examined. Their ages ranged from 12 to 61 years.

Subjects lay supine on a couch in a warm room. The arm under examination was supported by two slings ${ }^{\circ}$ approximately $90 \mathrm{~cm}$. in length suspended from a gantry $\vec{\circ}$ (Fig. 1). One sling was placed around the elbow and the second around the wrist so that the forearm could be $\vec{\omega}$ moved freely in the horizontal plane without touching ? the chest. The position of the head and shoulders was $\bar{\exists}$ carefully adjusted with pillows so that the subject was? comfortable and able to relax. A webbing cuff was also $y$ placed round the wrist. A nylon cord attached to this led iv over a pulley mounted on a pillar behind the heas, $\omega$ weights being placed on a hook on this line. The relatife positions of the pulley and the subject were adjusted $\$$ 으 that the cord was as nearly as possible parallel with the upper arm in both vertical and horizontal planes.

Maximal tension was measured by means of a spring balance attached to the cord, the subject being requesteg to extend the arm as steadily and forcefully as possibe, Ф the balance being used to keep the elbow joint at $90^{\circ}$. Theie $\vec{P}$ range of tensions found in the healthy subjects extende from 6.5 to $16 \mathrm{~kg}$. Next, trial weights were applied so that ${ }^{\circledR}$ the subject became accustomed to maintaining the elbow at a right angle while resisting the load of the weight. In healthy subjects and in most patients with dystrophy the contribution of the long head of the triceps to extension of the elbow at the tensions used appeared to be small. $\frac{\mathrm{D}}{\mathrm{Q}}$ The E.M.G. was therefore recorded from the lateral head $\varrho$ of the triceps. The bulkiest portion of the lateral head was $\overrightarrow{0}$ found by palpation and the needle inserted at this point. 3

Three Disa needles (type $13 \mathrm{~K} \mathrm{51}$ ) were used in the study. Their length was $30 \mathrm{~mm}$., outside diameter $0.45 \mathrm{~mm}$., core area $0.07 \mathrm{~mm}^{2}$, and bevel angle $15^{\circ}$ Before use each needle was prepared in $0.9 \%$ saline by applying 3V A.C. at $50 \mathrm{c} / \mathrm{s}$ across core and shaft with a suitable meter in series. A minimal impedance of 2.5 to 7 kilohms was usually reached within 15 sec., after which no further fall occurred. If the impedance did not fallo rapidly on application of current the needle was wiped $₹$ firmly with a sterile swab and the process repeated. The $\mathrm{o}$ needle led through its standard cable and socket to a pair of cathode-follower input stages mounted on the couch $\frac{7}{0}$ close to the subject's arm. The screen of the cable was earthed and an earth lead was taken to a metal plate $N$ $3.5 \mathrm{~cm}$. $\times 6 \mathrm{~cm}$. strapped to the forearm. The core and shaft of the needle were each connected via $0.15 \mu \mathrm{F}$ 


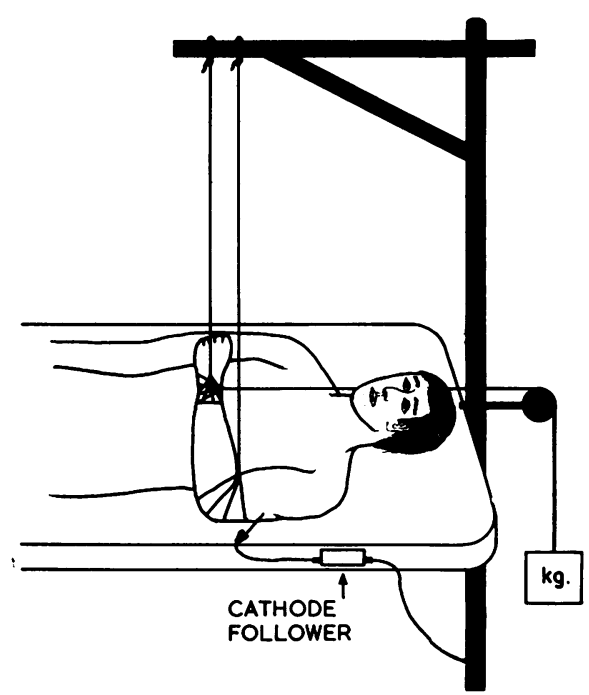

FIG. 1. Position of subject, slings, and cathode follower.

capacitors to the grids of the double triode used in the cathode-follower stages. The input impedance of each half to earth was approximately equivalent to 75 megohms with $7 \mathrm{pF}$ in parallel. The output impedance of the cathode-follower was $2.5 \mathrm{kilohms}$ and long leads were taken to a Tektronix FM 122 pre-amplifier and 502 oscilloscope. The frequency response of the pre-amplifier was $3 \mathrm{db}$ down at $40 \mathrm{Kc} / \mathrm{s}$ and $8 \mathrm{c} / \mathrm{s}$ and the amplification was approximately 1,000 times. A calibration signal of $1 \mathrm{mV}$ derived from the oscilloscope was always applied to the input of the cathode-follower at the end of each recording. A time scale of pulses at $10 \mathrm{msec}$. intervals was derived from a crystal-controlled oscillator and frequency divider and this was displayed on the lower beam of the oscilloscope, except in a few earlier experiments when a $50 \mathrm{c} / \mathrm{s}$ signal was used. Continuous records were taken on moving film at approximately $30 \mathrm{~cm}$. $/ \mathrm{sec}$. with a stationary spot. A separate oscilloscope and loud speaker were used to monitor the activity of the muscle.

The needle was inserted into the muscle and records for measurement of the activity-tension curve were taken with four standard weights of $0.5,1.0,1 \cdot 5$, and $2.0 \mathrm{~kg}$. Care was taken not to allow the forearm or the needle to move so that records were taken at the same muscle length from the same site. Following this, recordings were made with the needle in other positions.

For analysis the film was projected on to the baseboard of a $35 \mathrm{~mm}$. enlarger and examined with the special counting device. Using this, the wave form is traced visually with a transparent straight edge which is free to move backwards and forwards on the enlarger baseboard (i.e., in the $\mathrm{Y}$ axis). A piece of fine-toothed rack is connected to the cursor through a sliding joint which provides backlash equivalent to an amplitude of $100 \mu \mathrm{V}$ on the trace. This rack does not therefore follow movements of the cursor which are less than the equivalent of $100 \mu \mathrm{V}$. When the cursor is moved a distance greater than this a pulse counter is operated once and the extent of the movement of the rack is recorded on a pair of rotary ratchet counters by means of a series of gears and levers. The ratchet counters are arranged so that the displacement in each direction is totalled separately.

A portion of trace from a healthy subject is shown in Fig. 2, the direction of analysis being from left to right. The interrupted lines indicate the movements of the cursor which occur without movements of the rack and show the backlash of $100 \mu \mathrm{V}$. At each transition from an interrupted line to a solid line a count is registered on the pulse counter. At the same point the measurement of amplitude is started and it ceases at the end of the movement of the cursor in that direction. In the epoch shown here the pulse counter would register nine counts and the ratchet counters would register the amplitude of five excursions in an upward direction and four excursions downwards. In the calculation of mean potential amplitude the total amplitude is divided by the number of counts in the same portion of the record and it is clear from Fig. 2 that $100 \mu \mathrm{V}$ must be added to this figure as a correction for the backlash.

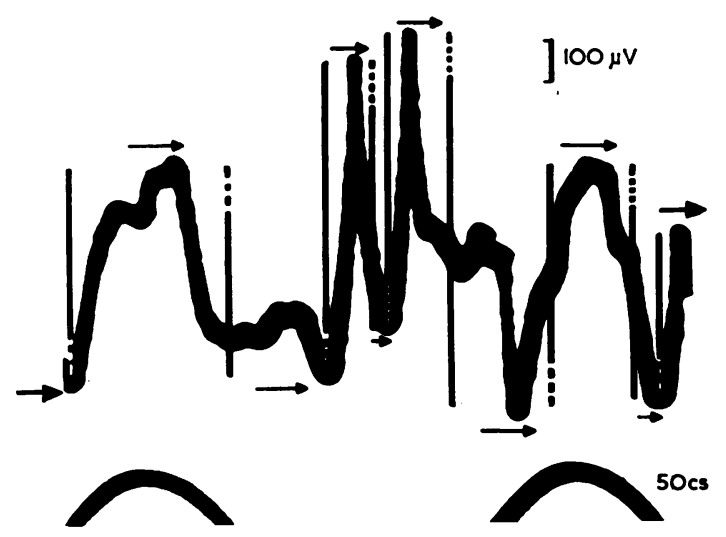

FIG. 2. Tracing from triceps in a healthy subject. Direction of analysis indicated by arrows. Interrupted lines indicate backlash of counter, solid lines show portions included in amplitude count (by permission of the Editors, Journal of Physiology).

In both healthy subjects and patients single sections of record one second in duration and free from artefact were examined, and the count rate and mean amplitude, expressed in $\mathrm{mV}$, were calculated. Patients with muscular dystrophy who were too weak to maintain steady tensions were excluded from the study as the analysis of electrical activity recorded during fluctuating effort was unsatisfactory; furthermore very weak patients often showed trick movements. For example, the contribution of the long head of the triceps to elbow extension might be substantially greater than is normally the case. From the practical point of view severely affected patients do not require this form of analysis as gross and easily recognizable changes in motor unit potential wave form are apparent on inspection of the trace. 
RESULTS

ACTIVITY-TENSION RELATIONSHIP IN HEALTHY SUBJECTS The relationship between electrical activity and tension was determined in 22 control subjects. In each case the needle was placed in the triceps at a point approximately $2 \mathrm{~cm}$. anterior to the junction of the lower third and upper two-thirds of a line joining the acromion to the lateral epicondyle of the humerus. At a depth of 2 to $2.5 \mathrm{~cm}$. a site could usually be found which showed clearly defined activity on the monitor CRT at $0.5 \mathrm{~kg}$. tension.

The four weights were applied in turn, recordings being made for a period of 2 to $3 \mathrm{sec}$. as soon as the activity had become uniform after the subject had taken up the load. The position of the forearm was carefully watched to ensure that the elbow remained at $90^{\circ}$.

The records were measured with the counter and the results are shown in Fig. 3 in which the count rate is plotted against tension. In several subjects activity-tension curves were determined on both sides and on different occasions. The curve for these subjects included in Fig. 3 is that which showed the highest count rate obtained at a tension of $2 \cdot 0 \mathrm{~kg}$.

The graph shows considerable variation from subject to subject; from the mean figures for the group as a whole (interrupted line) it is clear that there was a linear rise in count rate with increasing

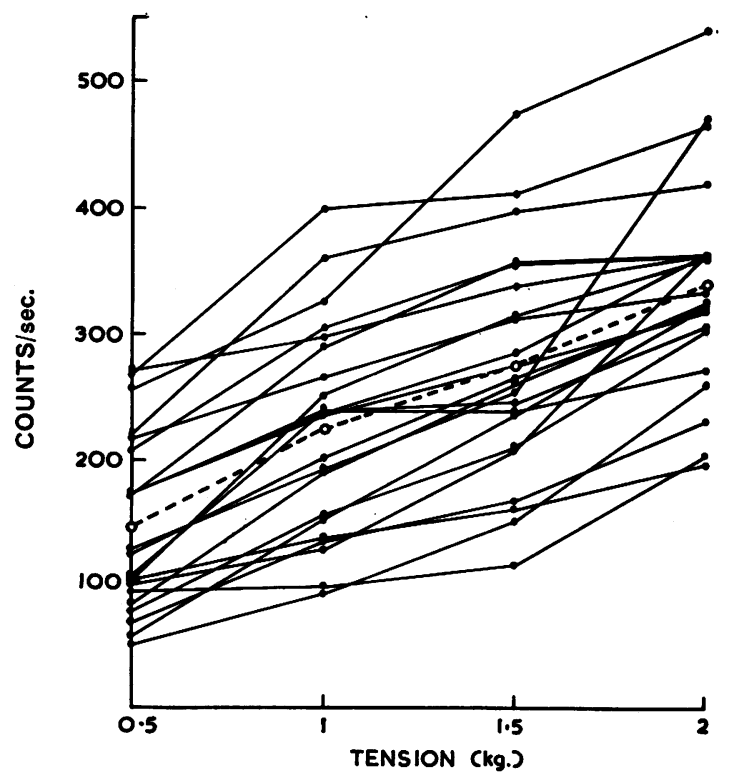

FIG. 3. Count rates of potential changes in triceps plotted against tension in 22 control subjects. Mean values indicated by interrupted line. tension. It will be noted that the projection of this line would not pass through the origin but would reach the baseline at a negative value of approxi mately $-0.5 \mathrm{~kg}$. This may be the reflection of the tension required to take up the slack in the muscles and in the cuff and sling. These activity-tension curves show that the upper figures for the group as a whole are $275,400,480$, and 543 counts/sec. for tensions of $0 \cdot 5,1 \cdot 0,1 \cdot 5$, and $2 \cdot 0 \mathrm{~kg}$. respectively.

READINGS AT $2.0 \mathrm{KG}$. IN HEALTHY SUBJECTS The variation between subjects was more fully examined in 23 healthy subjects. At $2.0 \mathrm{~kg}$. tension the needl£ was placed in different positions in the muscles, the depth varying from 2.75 to $1.5 \mathrm{~cm}$. During contrac $\vec{D}$ tion the trace was observed in order to find the areas showing the greatest activity. Because the counting" had to be carried out after the observations had been made it was necessary to make numerous photo-graphic records in order to be sure that the highest figure was measured. Even with practice it was not possible to estimate the count rate from the oscil? loscope and loudspeaker to within 50 counts/sec., thoi error sometimes being considerably higher than this iv The activity during a period of one second wisis examined using the counter. The count rates tained are shown in Fig. 4 where they are plottig $\mathrm{d}$ 음 vertically above the index number of the subject. $\Phi_{n}-$ order to determine whether the readings in individuin subjects are representative of the group as a whdie an analysis of variance has been made of these data. The estimate of variance between samples is 175 within samples 2572. The ratio of variance is 6.8 a using Snedecor's variance ratio test, this indicates. ${ }^{+}$ that the samples from the individual subjects show significantly less variation than the population as a whole $(F=2.0$ at $1 \%$ level, $\operatorname{df}(\nu 1)=115, \operatorname{df}(\nu 2)=\bar{\partial}$ 22).

The count rates at $2.0 \mathrm{~kg}$. tension may be used to define the upper portion of the normal range. Thus $\overrightarrow{\vec{A}}$ the highest figure is $543 / \mathrm{sec}$., the only count over $500 / \mathrm{sec}$., whereas five subjects had counts between $\bar{\partial}$ 450 and $500 / \mathrm{sec}$. The highest count at $2.0 \mathrm{~kg}$. tension was found in 11 out of 21 instances at the first needle insertion when the activity at four tensions was examined. In 10 other subjects a higher figure was found during the subsequent search for areas show -3 ing greatest activity at $2 \cdot 0 \mathrm{~kg}$.

RESULTS IN PATIENTS WITH MUSCLE DISEASE Eighteen patients suffering from muscle disease were studied. They included eight suffering from progressive muscular dystrophy, five with dystrophia myotonica, and five with polymyositis. The techniques were $N$ similar to those used in the control series, the triceps $N$ muscle and the same standard weights being used for 


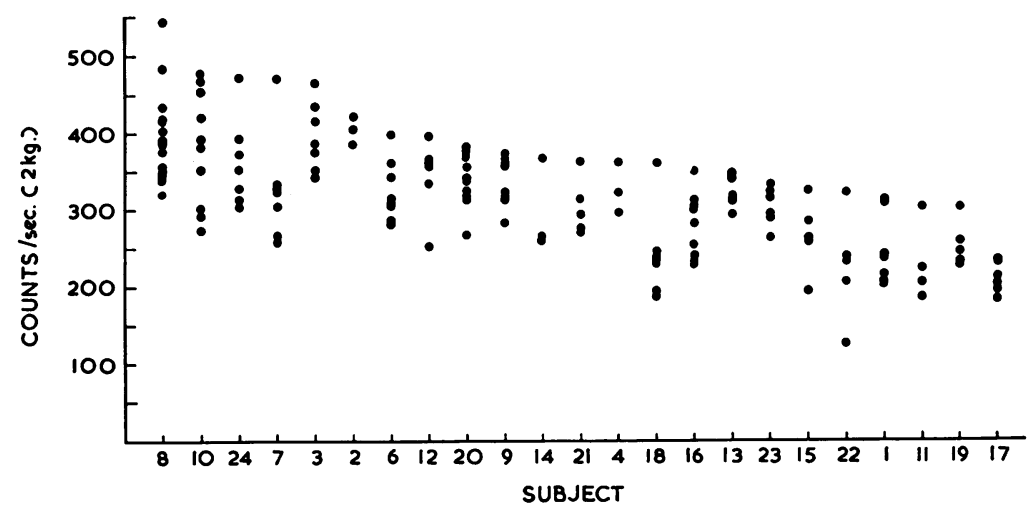

FIG. 4. Count rates of potential changes at $2.0 \mathrm{~kg}$. tension in triceps with different needle positions in 23 control subjects.

the activity-tension curves. Triceps was by no means the weakest muscle in many of the patients but, since preliminary observations had shown that count rates varied considerably in different muscles, it was clear that valid comparisons between healthy subjects and patients could only be made by using the same muscle in both groups.

Activity-tension curves were estimated in 18 patients. Illustrative records from a healthy subject and a patient with muscular dystrophy are shown in Figure 5 . In this case there is an obvious difference in the number of potential changes, which is confirmed by the count rates shown below each trace. The figures relating to mean amplitude will be discussed in a later section.

Results from the 18 patients are shown graphically in Fig. 6 in which the normal range illustrated in Fig. 3 is shown as a shaded area. Count rates up to nearly three times the highest normal figure were obtained in some patients. In all but three patients the curves lie outside the normal range in whole or in part. The increase in count rate is most conspicuous at $2.0 \mathrm{~kg}$. tension so that this appears to provide the most useful level of activity for differentiating between healthy subjects and patients.

The highest count rate at this tension was $1,452 / \mathrm{sec}$. obtained from a patient, Mrs. A.Y. (NH no. A15511), who was 56 years of age and had suffered from slowly progressive muscular dystrophy of facio-scapulohumeral type for over 30 years. The maximal tension which she was able to sustain was $4 \mathrm{~kg}$. (normal range 6.5 to $16 \mathrm{~kg}$.). Thus her effort at $2.0 \mathrm{~kg}$. tension was half-maximal compared with the healthy subjects in whom $2.0 \mathrm{~kg}$. tension is between one-third and one-eighth of maximum. The four patients who had counts over $1,000 / \mathrm{sec}$. at $2.0 \mathrm{~kg}$. tension showed maximal tensions of $4 \mathrm{~kg}$. or less; at the other end of the range there were six patients with count rates of over $600 / \mathrm{sec}$. whose maximal effort was within the normal range of 6.5 to $16 \mathrm{~kg}$., so that the relation

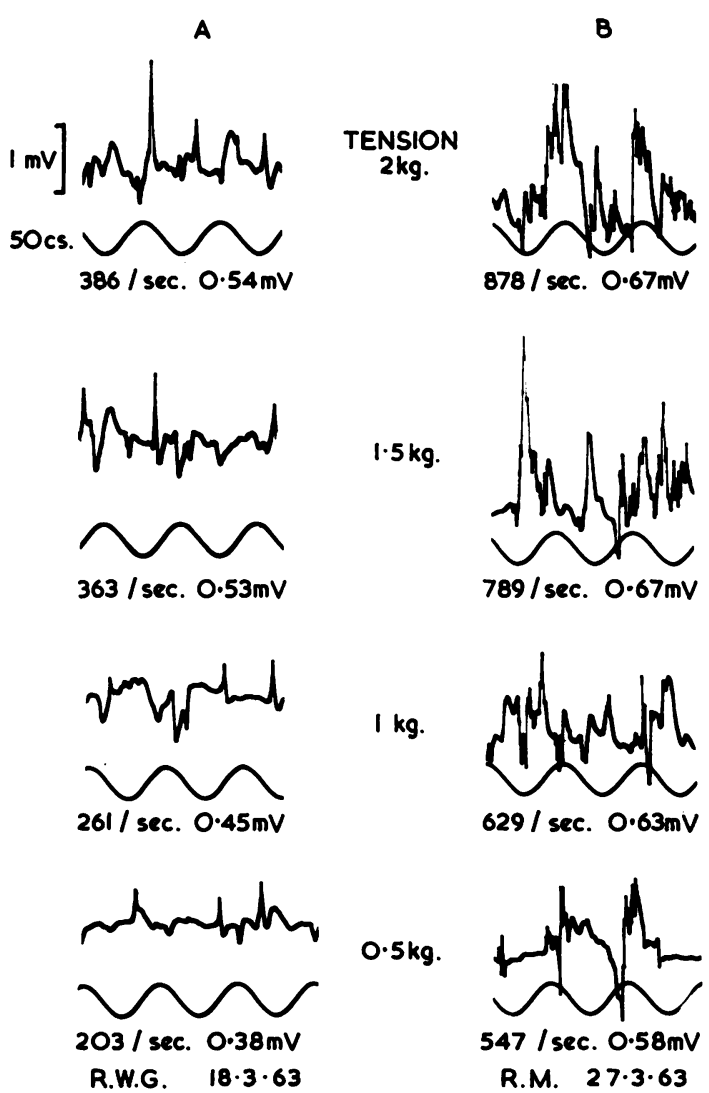

FIG. 5. Portions of primary trace from triceps in healthy subject $(A)$ and patient with progressive muscular dystrophy (B) with count rates and mean amplitudes at four standard tensions. The count rates are higher in the patient than in the healthy subject and confirm the visible difference. 


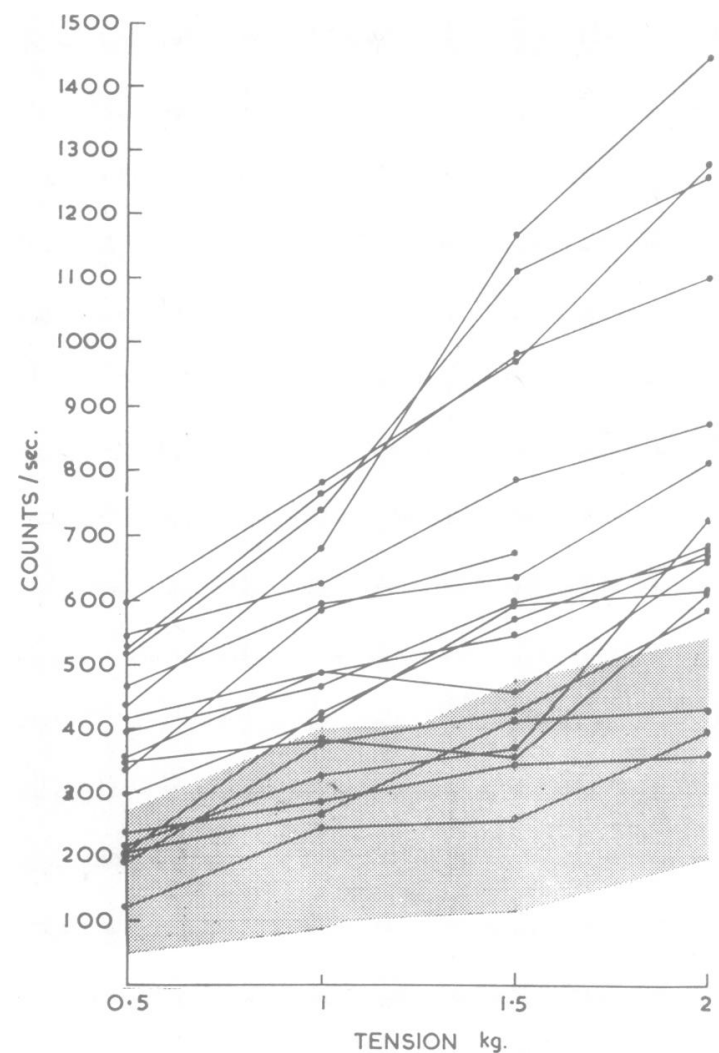

FIG. 6. Count rates of potential changes in triceps plotted against tension in 18 patients with muscle disease. Shaded area indicates normal range.

between low maximal tension and high count rates is approximate rather than precise.

MEAN AMPLITUDE OF POTENTIAL CHANGES The mean amplitude of potential changes is calculated by dividing the total amplitude of all excursions expressed in $\mathrm{mV}$ by the total count for the same portion of the record. Figure 7 shows 22 activity-tension curves in the healthy subjects in which mean amplitude, expressed in $\mathrm{mV}$, is plotted against the tension in kilograms. These figures represent the mean amplitudes corresponding to the count rates shown in Figure 3. The scatter is wide but the mean values for the group are $0.35,0.40,0.47$, and $0.51 \mathrm{mV}$ at tensions of $0.5,1.0,1.5$, and $2.0 \mathrm{~kg}$. respectively. It is clear that there is a slight rise in mean amplitude for the group with increasing tension but that the percentage increase in the amplitude is less than the percentage increase in mean count rate $(45 \%$ as opposed to $138 \%$ over the tension range examined). On examination of individual curves in Fig. 7 there

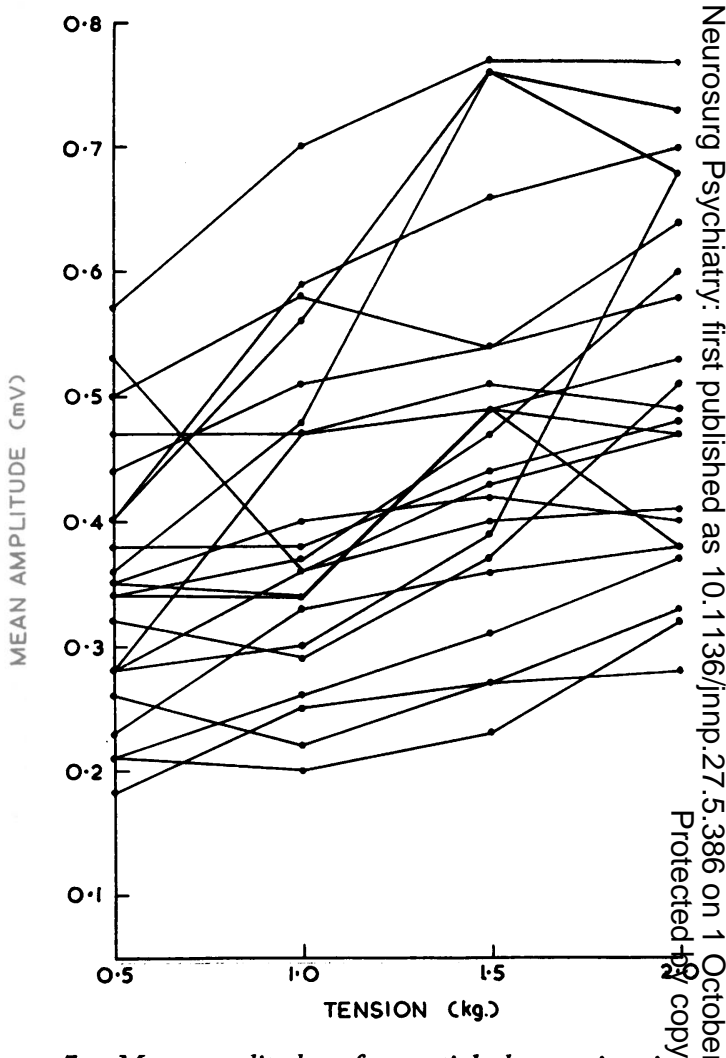

FIG. 7. Mean amplitudes of potential changes in tricesps plotted against tension in 22 control subjects. Theses
correspond to the curves shown in Figure 3 .

are obvious irregularities, the mean amplitude falling markedly with increasing tension in several instances $\frac{-}{0}$ Such exceptions to the general behaviour may be due to slight movement of the recording electrode or to alterations in the pattern of activity at the needle tip caused by the change in tension. In one subject in whom the mean amplitudes were $0.53 \mathrm{mV}$ and $0.36 \mathrm{mV}$ at $0.5 \mathrm{~kg}$. and $1.0 \mathrm{~kg}$. tension respectivelyo the count rates were $101 / \mathrm{sec}$. and $254 / \mathrm{sec}$. Examination of the records reveals the presence of a number of small potentials in the record at $1.0 \mathrm{~kg}$. These were not seen at the lower tension which contained fewer oscillations of greater amplitude, a single unit close to the needle making a major contribution to the trace; thus the mean amplitude was higher at the lower count rate. In only five instances were there inconsistent falls of mean amplitude between twe tensions greater than $10 \%$ of the figure at the lowere. tension. It is interesting that these accompanied count rates of $360 / \mathrm{sec}$. or less. If the combination of small needle movements and sampling error is 


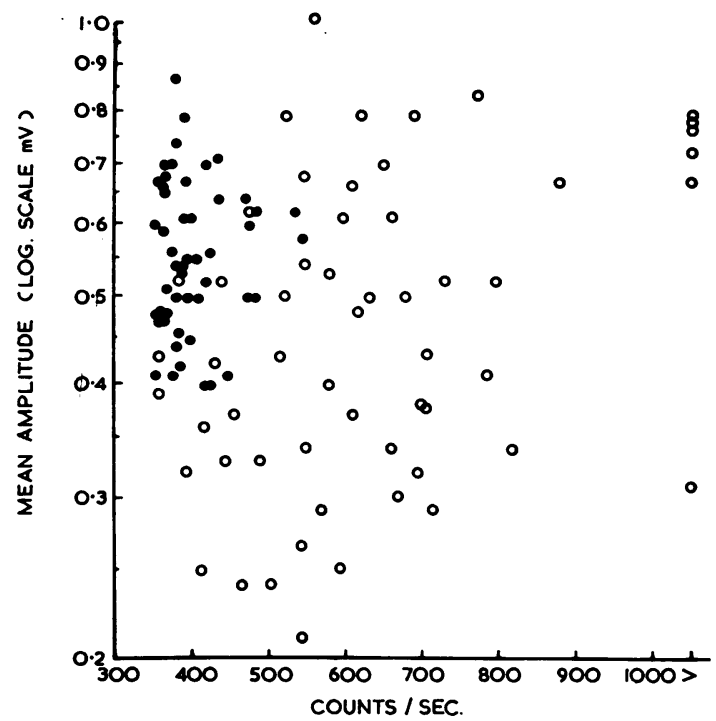

FIG. 8. Mean amplitudes (log scale) at $2.0 \mathrm{~kg}$. tension in triceps plotted against count rates over 350/sec. found in 23 healthy subjects (closed circles) and 18 patients with muscle disease (open circles).

responsible for these irregularities it is reassuring to find that except in certain cases with low count rates these factors will not produce inconsistent results.

The relationship between mean amplitude and count rate is shown in Fig. 8 for all counts over $350 / \mathrm{sec}$. at $2.0 \mathrm{~kg}$. tension. Each figure is calculated from measurements of a one-second period of activity; 23 control subjects (closed circles) and 18 patients with muscle disease (open circles) are shown. It is clear that the affected patients show a much wider range of amplitude $(0.21$ to $1.0 \mathrm{mV})$ than the healthy subjects in whom the figures range from 0.40 to $0.87 \mathrm{mV}$.

The traces seen in some patients with muscle disease which show high count rates and high mean amplitudes are of great interest because the large mean amplitude implies that active subunits are close to the tip of the recording needle, probably within $0.5 \mathrm{~mm}$. or less (Buchthal, Guld, and Rosenfalck, 1957). The high count rate also indicates that many units near to the tip of the needle are active because only potential changes over $100 \mu \mathrm{V}$ are counted and distant activity would be attenuated below this level. A specimen of record in a patient with polymyositis (Mrs. J.L. MH no. K21810) is illustrated in Fig. 9 to demonstrate the large amplitude of individual potentials. This, in association with the brief rise time, suggests that there was profuse activity near the needle. The mean amplitude in this muscle was $0.79 \mathrm{mV}$ with a count rate of

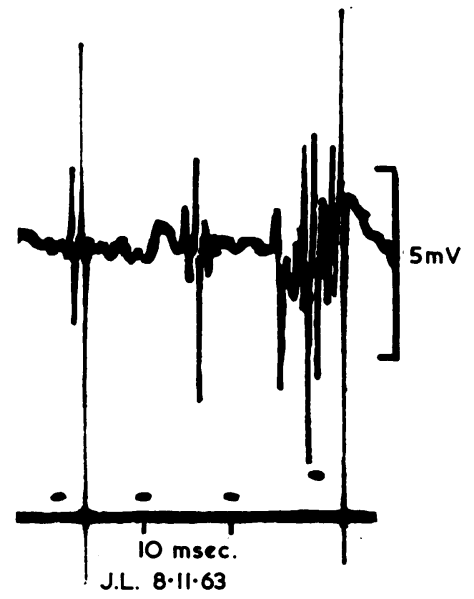

FIG. 9. Portion of primary trace in patient with dermatomyositis to show large amplitude, brief rise time, and high count rate of the potential changes.

$1,286 / \mathrm{sec}$. This record also shows that the brevity of the potential changes makes it possible for high counts to be reached in spite of their large amplitude, as suggested by Buchthal and Rosenfalck (1963).

At the lower end of the range of mean amplitude there are 24 readings in eight patients which are below those encountered in healthy subjects. Half of these have count rates within the normal range. This combination of findings is frequently encountered in severely affected muscle. The explanation of this is clarified by consideration of a patient whose weakness was so extreme that he was not included in Figure 8. This was a 61-year-old man (Mr. A. F. NH no. A9450) with progressive muscular dystrophy of 35 years' duration. The muscle was so weak that movement of the joint was only just possible. A portion of the record is shown in Fig. 10 from which it can be seen that individual potential changes are of low amplitude and many are of brief duration. Table I shows the figures obtained. Many potentials were below $100 \mu \mathrm{V}$ in amplitude and so were only counted when the significance level was reduced, the count rate being highest and the mean amplitude lowest when potentials of $25 \mu \mathrm{V}$ and above were counted. It can be envisaged that in an advanced case not only would the amplitude of individual units be low but even the number would be reduced as it is generally acknowledged that this occurs in the late stages of the disease. Thus low count rates and low mean amplitudes may be expected in the most severely affected patients. In such patients counting at levels lower than $100 \mu \mathrm{V}$ is necessary if a realistic picture of electrical activity is to be obtained.

From the data presented in Fig. 8 it is clear that patients with myopathy may be distinguished from healthy subjects by the occurrence of high count rates or abnormal mean amplitudes which may be 


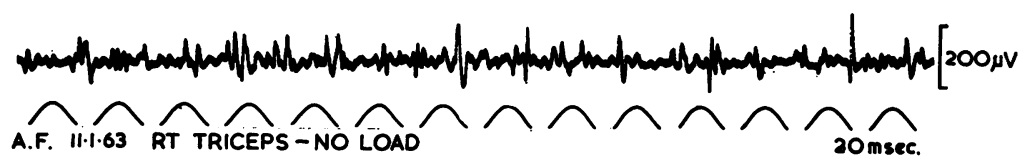

FIG. 10. Portion of primary trace in patient with severe progressive muscular dystrophy. Triceps was severely affected (M.R.C. scale 2) and this record was taken at maximal effort. Count rates and mean amplitudes are shown in Table I.

TABLE I

COUNT RATES AND MEAN AMPLITUDE AT THREE LEVELS IN A PATIENT WITH SEVERE WEAKNESS (PRIMARY TRACE SHOWN IN FIG. 10)

\begin{tabular}{rcc}
$\begin{array}{l}\text { Count } \\
\text { Level } \\
(\mu V)\end{array}$ & Counts/Second & $\begin{array}{l}\text { Mean } \\
\text { Amplitude } \\
(m V)\end{array}$ \\
\hline 100 & 72 & $0 \cdot 12$ \\
50 & 157 & $0 \cdot 08$ \\
25 & 335 & $0 \cdot 04$
\end{tabular}

above or below the normal range. There remain eight readings encountered in three patients in which both count rate and mean amplitude are within the normal range. However, in each of these patients there were other areas in the muscle which were abnormal by the criteria already described so it proved possible to delineate every patient from the control group. This illustrates the advantage of recording from more than one site.

\section{DISCUSSION}

In clinical electrodiagnosis previous attempts to analyse the pattern of activity on voluntary effort have been directed towards automatic methods such as frequency analysis (Walton, 1952; Richardson, 1951). In physiological studies Bergström (1959) compared spike counts with the integrated activity, and Close, Nickel, and Todd (1960) used a pulse counter to count spikes during voluntary effort against tension, but such methods do not yet appear to have been applied to the study of diseased muscle.

The method used in the present study is designed to circumvent some of the unknown factors which may influence automatic frequency analysis or pulse counting using a conventional counter. Thus the present technique depends upon following the trace, using the point of change of direction of potential changes as the index mark for assessing the amplitude of the following potential; this overcomes the difficulty inherent in using a fixed baseline. In practice both pulse counters and integrating devices are influenced by the voltage level which depends on the coupling capacitances in the system. A conventional RC coupled pulse counter will fail to count small oscillations superimposed on slower and larger waves unless the coupling time constant is reduce $\overrightarrow{\mathrm{F}^{*}}$ to a value which may interfere with the transmission of slow changes.

The relationship between potential changes counted by this method and the action potentials of individual motor units requires consideration because all the records described in the paper have beerf? made at levels of voluntary activity at which it is not possible to identify the action potentials of individuat $L$ units. It might be thought that each potential change represented one phase of an action potential but if this was so the amplitude should remain constan with tension. Figure 7 shows that mean amplitudeiv increases with rising tension, as does the count rateir This suggests that summation of the activity $-8 \mathrm{ft}_{0}$ different units may occur by coincidence in time potential changes in the same direction. No dotsp some cancellation also occurs when potentials of opposite sign are simultaneous but, if cancellation were dominant, mean amplitude might be expeçof to decrease with increasing tension. The precise co figuration of the waveforms of individual units me be expected to influence this complex interaction follows that we may consider potential changes as representing motor unit potentials only in a generaP way, summation and cancellation obscuring a cleas relationship. Thus it is incorrect to refer to th counts as action potential counts, and the count rates. described in this study should not be regarded as having a definite relation to the discharge rate of motor units.

In order to measure the relation between count rate and tension some of the sources of variation? such as the particular muscle examined, limb posture and hence muscle length, the type of needle used, and the high input impedance, were kept constant:Despite this it is clear that there was a considerable variation from subject to subject (Fig. 3) and that needle position (Fig. 4) affected the count rate. Larges electrodes might sample a greater number of motor units but the use of a small recording electrode is necessary if patchy involvement of muscle is to be detected in polymyositis. It is of interest in this con 근. nexion that the highest potential counts recorded inf the soleus by Close, Nickel, and Todd (1960) were of the order of $200 / \mathrm{sec}$. using wire electrodes bared fo్ㅠ 
$\frac{1}{2}$ in. at the tip. These were taken at maximal tension, and, assuming that the pulses recorded by them were monophasic, this figure becomes $400 / \mathrm{sec}$. in terms of potential changes measured by the present method. It may be that small needle electrodes give higher count rates for a given tension than large electrodes and this point requires further examination, but their method of counting differed considerably from the technique described here and only approximate comparisons may be made.

If there is significant summation and cancellation of action potentials it might be expected that the briefer the individual components, the more likely it is that they will appear separately and be counted. Thus at maximal effort we might expect higher counts in patients with muscle disease than in healthy subjects because the action potential duration is reduced in muscle disease and polyphasic potentials are more frequent. It is also probable that individual motor units develop less tension than in healthy subjects (Lenman, 1959). Therefore the examination of activity at low tensions would be expected to bring out a difference between the density of active units in the region of the needle in healthy and affected muscles. The use of a standard tension of $2.0 \mathrm{~kg}$. suitable to the triceps allowed comparisons to be made between subjects whereas maximal tension varied so widely that it was not a useful standard.

Although it has been said that increase in mean amplitude is a result of summation of coincident action potential phases in the same direction, it is possible that motor units recruited at high tensions are of greater amplitude than those recruited at minimal effort (Norris and Gasteiger, 1955). The potentials of 10 to $15 \mathrm{mV}$ amplitude in Fig. 9 were only occasionally seen in records from the same region of the muscle taken at tensions less than $2.0 \mathrm{~kg}$. This question requires further investigation.

Some patients with muscle disease showed mean amplitudes less than those found in healthy subjects at $2.0 \mathrm{~kg}$. tension. The count rates in these patients were often within or close to the normal range. It is not clear whether motor unit potentials were smaller in this group than in those showing high mean amplitudes or whether potentials were attenuated by the presence of inactive tissue such as fat or connective tissue. The findings shown in Fig. 10 and Table I suggest that at least in some patients it seems likely that action potential amplitudes were smaller. Possibly the use of more selective electrodes would help to clarify the issue. If areas of very low activity were found in severely involved muscle it would seem likely that such were occupied by inactive tissue. With the electrodes used in the present study, areas showing little activity were found frequently even in patients showing high count ra:es in other areas. Thus it was always necessary to make a deliberate search for portions of muscle showing high count rates.

A disadvantage inherent in examining photographic records is the long delay before the count rate is known. It is not always possible to be certain when the areas showing high counts have been found, judging by the visual display and loudspeaker, and it may be necessary to carry out counts on the records from several areas in order to find the highest value. Even with experience it is not possible to make more than rough estimates of the count rate by judgment alone and this study has made it clear that subjective assessment is unreliable except in instances of gross alterations in the pattern.

Examination of the muscle could be made more thorough and more rapid with automatic methods of counting potential changes and measuring amplitude. Automatic methods would also help to extend studies to other muscles in healthy subjects and in patients with muscle disease. Certainly such studies are required, for it is not possible at the present time to investigate the weakest muscles in patients or the most suitable muscles in carriers of the sex-linked recessive forms of dystrophy.

The development of an electronic method of counting analogous to that used in the mechanical counter is in progress but there are several problems to be overcome in designing a system which will analyse both the most rapid and the slowest potential changes encountered in this study.

\section{SUMMARY}

A new method of analysing the rate and amplitude of potential changes in the triceps muscle has been used in 24 healthy subjects and in 20 patients with muscle disease. The method involved the examination of photographic records taken under standard conditions and at known tensions. The analysis is performed by counting the rate and mean amplitude of potential changes using a mechanical counting system. The reliability of the results is independent of the frequency and the amplitude of the recorded potentials. Counts up to three times the highest encountered in a healthy subject were seen in patients with muscle disease. Abnormalities in the mean amplitude of potential changes were also found. The significance of these changes is discussed.

I am most grateful to Professor R. W. Gilliatt and Mr. H. B. Morton for advice and encouragement and to the Medical Research Council for help with apparatus. A special grant from the Muscular Dystrophy Group for technical assistance and counting apparatus is also gratefully acknowledged. 


\section{REFERENCES}

Barwick, D. D. (1963). In Research in Muscular Dystrophy; Proc. 2nd Symposium, Muscular Dystrophy Group, pp. 10-19. Pitman Medical Publ. Co., London.

Bergström, R. M. (1959). The relation between the number of impulses and the integrated electric activity in electromyogram. Acta physiol. scand., 45, 97-101.

Buchthal, F., Guld, C., and Rosenfalck, P. (1957). Volume conduction of the spike of the motor unit potential investigated with a new type of multielectrode. Ibid., 38, 331-354.

- , and Rosenfalck, P. (1963). In Muscular Dystrophy in Man and Animals, edited by G. H. Bourne and M. N., Golarz, p. 197. Karger, Basel.

Close, J. R., Nickel, E. D., and Todd, F. N. (1960). Motor-unit action-potential counts. Their significance in isometric and isotonic contractions. J. Bone Jt Surg., 42A, 1207-1222.

Gilliatt, R. W. (1962). Electrodiagnosis and electromyography in clinical practice. Brit. med. J., 2, 1073-1079.

Kugelberg, E. (1949). Electromyography in muscular dystrophies. Differentiation between dystrophies and chronic lower motor neurone lesions. J. Neurol. Neurosurg. Psychiat., 12, 129-136.
Lenman, J. A. R. (1959). Quantitative electromyographic changes associated with muscular weakness. Ibid., 22, 306-310.

Norris, F. H., and Gasteiger, E. L. (1955). Action potentials of single $\subseteq$ motor units in normal muscle. Electroenceph. clin. Neuro-O physiol., 7, 115-126.

Pinelli, P., and Buchthal, F. (1953). Muscle action potentials in myopathies with special regard to progressive muscular $\square$ dystrophy. Neurology (Minneap.), 3, 347-359.

Richardson, A. T. (1951). The analysis of muscle action potentials in the differential diagnosis of neuromuscular diseases. Arch. phys. Med., 32, 199-206.

van den Bosch, J. (1963). In Research in Muscular Dystrophy, Proc. 2nd Symposium, Muscular Dystrophy Groups, pp. 23-30. Pitman. Medical Publ. Co., London.

Walton, J. N. (1952). The electromyogram in myopathy: Analysis with the audio-frequency spectrometer. $J$. Neurol. Neurosurg. Psychiat., 15, 219-226.

Willison, R. G. (1963). A method of measuring motor unit activity in human muscle. J. Physiol. (Lond.), 168, 35-36P. 